\title{
Lovastatin decreases acute mucosal inflammation via 15-epi-lipoxin $\mathrm{A}_{4}$
}

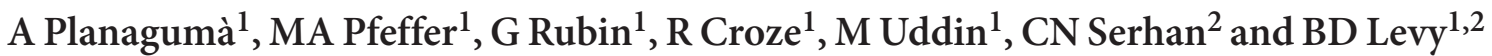

The widespread use of statins for hypercholesterolemia has uncovered pleiotropic anti-inflammatory properties that were unexpected based on the drugs' original design; yet, mechanisms for these protective actions remain uncertain. In this study lovastatin triggered biosynthesis of the anti-inflammatory and pro-resolving mediator 15-epi-lipoxin $\mathrm{A}_{4}$ (15-epi-LXA 4 ). During interactions between human neutrophils and airway epithelial cells, the statin-induced increase in 15-epi-LXA 4 was associated with increased 14,15-epoxyeicosatrienoic acid (14,15-EET) generation. When added to activated neutrophils, 14,15-EET enhanced 15-epi-LXA 4 biosynthesis. In a murine model of airway mucosal injury and inflammation, lovastatin increased 15-epi- $\mathrm{LXA}_{4}$ formation in vivo and markedly decreased acute lung inflammation. Administration of 15-epi-LXA 4 also inhibited lung inflammation in an additive manner with lovastatin. Together, these results indicate that statin-triggered 15-epi-LXA 4 generation during human leukocyte-airway epithelial cell interactions is an endogenous mechanism for statin-mediated tissue protection at mucosal surfaces that may also be relevant in the statins' ability to stimulate the resolution of inflammation.

\section{INTRODUCTION}

Statins are potent cholesterol-lowering agents with pleiotropic anti-inflammatory properties. ${ }^{1}$ Inhibition of 3-hydroxy3-methyl-glutaryl-CoA reductase does not explain all of these drugs' anti-inflammatory actions. ${ }^{1}$ Of interest, statins can regulate innate and adaptive immune responses, ${ }^{2,3}$ as well as endothelial and epithelial cell function. ${ }^{4-6}$ Prospective human clinical trials have provided strong evidence for statin-mediated anti-inflammation, as individuals with normal plasma cholesterol who were randomized to receive statin therapy had marked decrements in both C-reactive protein, which was monitored as a marker of systemic inflammation, and cardiovascular events. ${ }^{7}$ In several animal models of injury or inflammation, statins also show protective actions, including sepsis, ${ }^{8}$ rheumatoid arthritis, ${ }^{9}$ asthma, ${ }^{10}$ emphysema, ${ }^{11}$ and acute lung injury (ALI). ${ }^{12}$ However, the mechanisms remain to be identified.

Acute respiratory distress syndrome and ALI have an annual incidence in the United States of more than 200,000 adults and still carry a 1-year mortality of $>40 \% .{ }^{13}$ There is no specific medical therapy for these conditions. Resolution of acute respiratory distress syndrome and ALI is an active process that is characterized in part by clearance of polymorphonuclear leukocytes (PMNs) from the lung. The natural resolution of inflammation occurs through local biosynthesis of braking signals, such as lipoxins (LXs) and 15-epi-LXs (also known as aspirin-triggered LXs), at sites of inflamed tissue. ${ }^{14} 15$-epi-LXs belong to a new genus of lipid mediators that are agonists of resolution and regulate airway inflammation ${ }^{14,15}$ and ALI. ${ }^{16,17} 15$-epi-LXs are locally produced through cell-cell interactions between leukocytes and resident cells during multicellular host responses to injury, inflammation, and microbial invasion (reviewed in ref. ${ }^{14}$ ). These lipid mediators show diverse counter-regulatory actions in pico- to nanomolar amounts, including inhibition of PMN functional responses ${ }^{18,19}$ and interactions with the endothelium, ${ }^{20}$ as well as stimulation of mucosal epithelial bacterial killing ${ }^{21}$ and macrophage clearance of apoptotic PMNs. ${ }^{22}$ Statins show many of the same anti-inflammatory properties as 15-epi-LXs. Both inhibit leukocyte adhesion, regulate cytokine and chemokine expression, induce endothelial and inducible nitric oxide synthase, lower matrix metalloproteinase expression and activation, increase tissue inhibitor of matrix protease, and inhibit nuclear factor- $\kappa \mathrm{B}$ activation (editorial in ref. ${ }^{23}$ in ref. ${ }^{24}$ ) with the new property for 15 -epi-LXA 4 of also promoting active resolution. ${ }^{25}$

${ }^{1}$ Pulmonary and Critical Care Medicine Division, Department of Internal Medicine, Brigham and Women's Hospital and Harvard Medical School, Boston, Massachusetts, USA. '2 Center for Experimental Therapeutics, Anesthesia, Perioperative and Pain Medicine, Brigham and Women's Hospital and Harvard Medical School, Boston, Massachusetts, USA. Correspondence: BD Levy (blevy@partners.org)

Received 28 August 2009; accepted 24 December 2009; published online 3 February 2010. doi:10.1038/mi.2009.141 
15-epi-LXs were first described as arachidonic acidderived products generated during interactions between cyclooxygenase-2 (COX-2) and 5-lipoxygenase (5-LO). ${ }^{26}$ Aspirin-acetylated COX-2 converts arachidonic acid to 15$R$-hydroxyeicosatetraenoic acid that is transformed by 5 - $\mathrm{LO}$ to 15 -epi-LXs. ${ }^{26}$ In addition to aspirin-acetylated COX-2, cytochrome P450 enzymes (CYP450s) are also capable of catalyzing $15-R$-hydroxyeicosatetraenoic acid production from arachidonic acid. ${ }^{27}$ Moreover, CYP450s can also convert arachidonic acid to epoxyeicosatrienoic acids $(\mathrm{EETs})^{28}$ that have anti-inflammatory properties when present in micromolar quantities. ${ }^{29}$ EETs are further metabolized by soluble epoxide hydrolase (sEH) to their corresponding diols, dihydroxyeicosatrienoic acids. ${ }^{30}$ Soluble epoxide hydrolase inhibitors increase LX generation and decrease plasma levels of proinflammatory cytokines to promote resolution of inflammatory responses. ${ }^{31}$ In this study we report that statin-initiated 15-epi-LXA 4 generation proceeds through transcellular biosynthesis during human PMN-epithelial cell interactions that promotes resolution of ALI in the lung.

\section{RESULTS}

\section{Lovastatin increases 15-epi-LXA 4 formation during interactions between human PMNs and airway epithelial cells}

In the presence of lovastatin ( $1 \mu \mathrm{m}$ hydroxy acid), human PMNs co-incubated with tumor necrosis factor- $\alpha$-primed airway epithelial cells (Calu-3) generated significant amounts of 15-epi$\mathrm{LXA}_{4}\left(>2.82 \pm 0.62\right.$-fold than Calu- 3 alone $\left(705 \mathrm{pg} \mathrm{ml}^{-1}\right), P<0.05$ for $n=3$ independent experiments; Figure 1a). In the absence of PMNs, lovastatin did not augment 15-epi-LXA 4 production by the cytokine-primed Calu-3 cells (1.02 \pm 0.34 -fold change). In addition, there was no significant increase in 15 -epi-LXA 4 by incubations of cytokine-primed Calu-3 cells with PMNs in the absence of lovastatin (1.44 \pm 0.22 -fold change). Statin-triggered 15-epi-LXA 4 formation correlated with increasing PMN numbers relative to Calu-3 cells (Figure 1b). For purposes of comparison, 15-epi-LXA 4 generated at a cell ratio of 0:1 (PMNs: airway epithelial cells) was assigned a value of 1 (mean, $541 \mathrm{pg}$ per ml of 15-epi-LXA ${ }_{4}$ ) and changes in 15-epi-LXA 4 amounts were expressed as a fold change for each cell ratio tested (1:1 (1.6 \pm 0.19 -fold increase), 2:1 (2.8 \pm 0.85 -fold increase), and 5:1 (3.8 \pm 0.8 -fold increase); $P<0.05$ for $n=5$ independent experiments; Figure 1b). In addition to Calu- 3 cells, lovastatin-triggered 15-epi-LXA 4 formation during human PMN interactions with primary normal human bronchial epithelial (NHBE) cells differentiated at an air-liquid interface (5:1 ratio, PMNs:NHBE, $927 \mathrm{pg}$ per ml of 15 -epi-LXA 4 , mean for $n=4)$ and with the distal human airway epithelial cell line A549 (5:1 ratio, PMNs: A549, $2.14 \mathrm{ng}$ per ml of 15 -epi-LXA 4 , mean for $n=3$ ). In further experiments, Calu-3 cells were used principally for ease of culture and cost considerations. These results indicate that a statin can increase 15 -epi-LXA ${ }_{4}$ generation in human cells through transcellular biosynthesis and that interactions between cytokine primed-airway epithelial cells and PMNs can enhance this biosynthetic reaction.
Lovastatin is well characterized as an inhibitor of 3-hydroxy3-methyl-glutaryl-CoA reductase, but mevalonate $(100 \mu \mathrm{M})$ did not block the statin-triggered 15-epi-LXA 4 formation (Figure 1c), indicating that lovastatin's mechanism for 15 -epi-LXA ${ }_{4}$ generation is distinct from its regulation of isoprenoid metabolism. Although independent from exogenous mevalonate, the actions of lovastatin were shared with simvastatin, another lipophillic statin (Figure 1c). Of interest, the broad-acting LO inhibitor, nordihydroguaiaretic acid, significantly inhibited lovastatin-triggered 15 -epi-LXA 4 formation by over $80 \%$ (Figure 1c), indicating that LO activity is critical to the compound's biosynthesis.

To identify what biosynthetic enzymes for LXs and 15-epi-LXs are expressed in these cell types, we next determined gene expression using semi-quantitative reverse transcriptase-PCR for sEH, 15-LO-1, 15-LO-2, and 5-LO (Figure 1d), as well as the related genes $5-\mathrm{LO}$-activating protein, $12-\mathrm{LO}$, and COX-2 in Calu-3 cells, Calu-3 cells exposed to tumor necrosis factor$\alpha\left(1 \mathrm{ng} \mathrm{ml}^{-1}, 24 \mathrm{~h}\right)$, freshly isolated human PMNs, and NHBE cells exposed to interleukin-13 (10 $\left.\mathrm{ng} \mathrm{ml}^{-1}, 96 \mathrm{~h}\right)$. sEH mRNA was present in all three cell types. In contrast, 15-LO-1 and 15-LO-2 mRNAs were only expressed in cytokine-primed NHBE cells, and 5-LO was only expressed in PMNs and Calu3 cells (Figure 1d). 5-LO-activating protein was only present in PMNs, and low levels of 12-LO mRNA were present in freshly isolated PMNs from peripheral blood (Supplementary Figure S1 online), indicating likely minor platelet contamination. ${ }^{32}$ Increased COX-2 mRNA expression was observed in only cytokine-primed airway epithelial cells (Supplementary Figure S1 online). Together, these findings indicate that neither cell type alone contains the genes required for 15-epi$\mathrm{LXA}_{4}$ biosynthesis; thus, providing a rationale for enhanced statin-triggered 15 -epi-LXA 4 generation through transcellular biosynthesis during cell-cell interactions.

\section{Lovastatin increases 14,15-EET generation}

To identify potential biosynthetic intermediates in lovastatintriggered 15-epi-LXA 4 formation, lipid extracts from PMN and airway epithelial cell cultures (5:1, PMNs:Calu-3) were analyzed using reversed-phase high-performance liquid chromatography (HPLC). In addition to 15-epi-LXA 4 (Figure 2a), statin-exposed cells also had a significant increase in materials with the retention time of 14,15-epoxyeicosatriene (14,15-EET) by charged aerosol detection (Figure $\mathbf{2 b}$ ). The statin-mediated increase in both 14,15-EET and 15-epi-LXA 4 was markedly increased by the sEH inhibitor 12-(3-adamantan-1-yl-ureido)-dodecanoic acid (AUDA) (Figure 2a,b). The actions of AUDA on statintriggered 15-epi-LXA ${ }_{4}$ formation were concentration dependent between 0.01 and $1 \mu \mathrm{M}$ (Figure 2c). AUDA $(0.01-1 \mu \mathrm{M})$ consistently increased the amount of 14,15-EET 10-fold higher than 15-epi-LXA 4 (Figure 2d).

\section{Exogenous addition of 14,15-EET increases 15-epi-LXA biosynthesis}

To determine whether the increased 14,15-EET reflected a role in statin-initiated transcellular 15-epi-LXA 4 biosynthesis, PMNs alone were activated in the presence of exogenous 14,15-EET. 

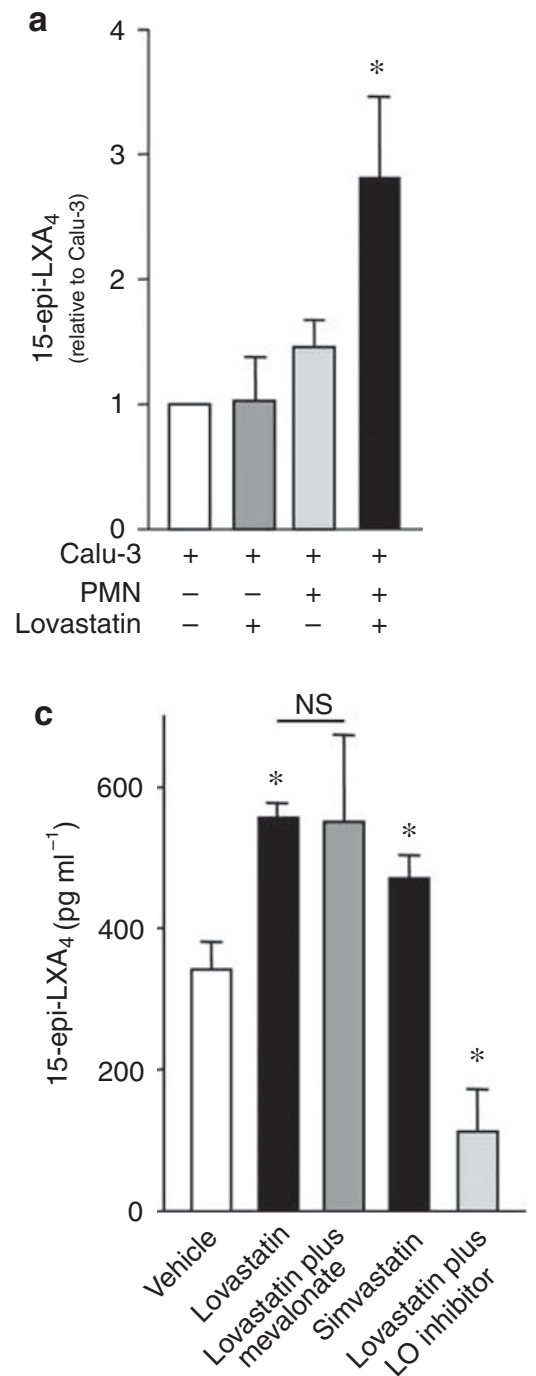

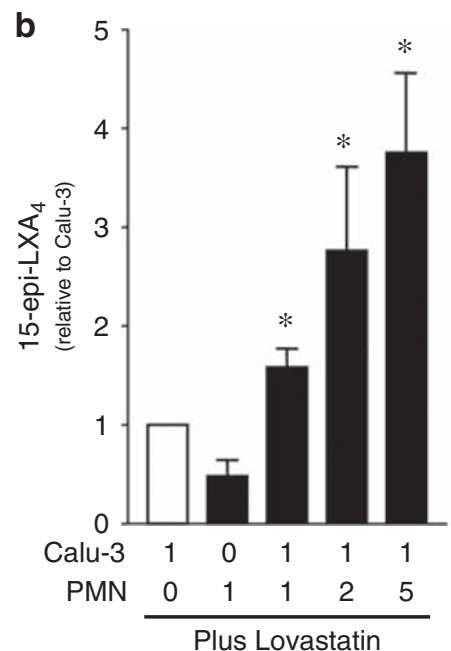

d

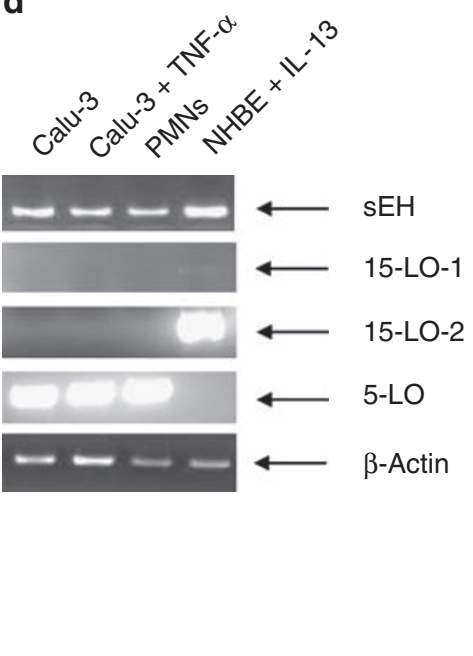

Figure 1 Lovastatin triggers 15-epi-lipoxin $\mathrm{A}_{4}$ (15-epi-LXA ${ }_{4}$ ) biosynthesis by activated human airway epithelial cells and polymorphonuclear leukocytes (PMNs). (a) 15-epi-LXA 4 levels were determined after Calu-3 cells were exposed to tumor necrosis factor- $\alpha\left(\mathrm{TNF}-\alpha ; 1 \mathrm{ng} \mathrm{ml}{ }^{-1}, 24 \mathrm{~h}\right.$ at $\left.37^{\circ} \mathrm{C}\right)$ and then to human PMNs in the presence or absence of lovastatin $\left(1 \mu \mathrm{m}, 30 \mathrm{~min}\right.$ at $\left.37^{\circ} \mathrm{C}\right)$ (see Methods). (b) Dose-dependent relationship between

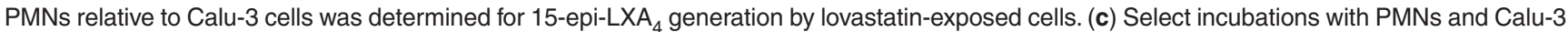
cells $(5: 1$ ratio) were carried out in the presence of lovastatin $(1 \mu \mathrm{m})$, mevalonate $(100 \mu \mathrm{m})$, simvastatin $(10 \mu \mathrm{m})$, nordihydroguaiaretic acid (NDGA; LO inhibitor, $5 \mu \mathrm{M})$, or vehicle $(0.1 \%$ ethanol). (d) Representative reverse transcriptase-PCR (RT-PCR) for pivotal lipoxin biosynthetic genes in Calu- 3 cells (without and with exposure to TNF- $\alpha$ ), freshly isolated human PMNs, and normal human bronchial epithelial (NHBE) cells exposed to interleukin-13 (IL-13; $10 \mathrm{ng} \mathrm{ml}^{-1}, 96 \mathrm{~h}$ at $\left.37^{\circ} \mathrm{C}\right)$. Results are expressed as the mean \pm s.e.m. for $\geqslant 3$ independent experiments. ${ }^{*} P<0.05$ using analysis of variance (ANOVA), NS, not significant.

No epithelial cells or lovastatin were present in these incubations. When exposed to exogenous 14,15-EET and activated with the divalent cation ionophore A23187, PMNs generated substantial amounts of 15 -epi-LXA 4 (Figure 3a). There was no significant production of 15 -epi-LXA 4 by PMNs activated in the absence of 14,15-EET. The effects of 14,15-EET on 15epi-LXA 4 generation in this system were regioselective and not shared by the related isomers 5,6-EET, 8,9-EET, or 11,12-EET (Figure 3b). There was a bell-shaped dose-response relationship between 14,15-EET and 15-epi-LXA 4 generation with a maximal response at 100 pmole (Figure 3c). Amounts of 14,15 -EET $>100$ pmole yielded a submaximal response; however, this decreased response was not statistically significant.
In contrast, significant changes in leukotriene $\mathrm{B}_{4}\left(\mathrm{LTB}_{4}\right)$ levels in these same incubations were only observed at doses of 14,15EET $>100$ pmole (Figure 3d), suggesting that the increase in 15-epi-LXA 4 with 14,15-EET was not secondary to decreased 5- $\mathrm{LO}$ conversion of arachidonic acid to $\mathrm{LTB}_{4}$. In addition, the omega oxidation products of $\mathrm{LTB}_{4}$ and 15 -epi-LXA 4 were not significantly altered by addition of the EET.

The selective 5-LO inhibitor, AA861, and the CPLA, inhibitor, methyl arachidonyl fluorophosphonate, both inhibited EET-stimulated 15-epi-LXA ${ }_{4}$ and $\mathrm{LTB}_{4}$ formation. In contrast, a CYP450 enzyme inhibitor, 17-octadecynoic acid, increased the amount of both 15-epi-LXA 4 and $\mathrm{LTB}_{4}$ in incubations with PMNs and 14,15-EET, likely secondary to this compound's 
a
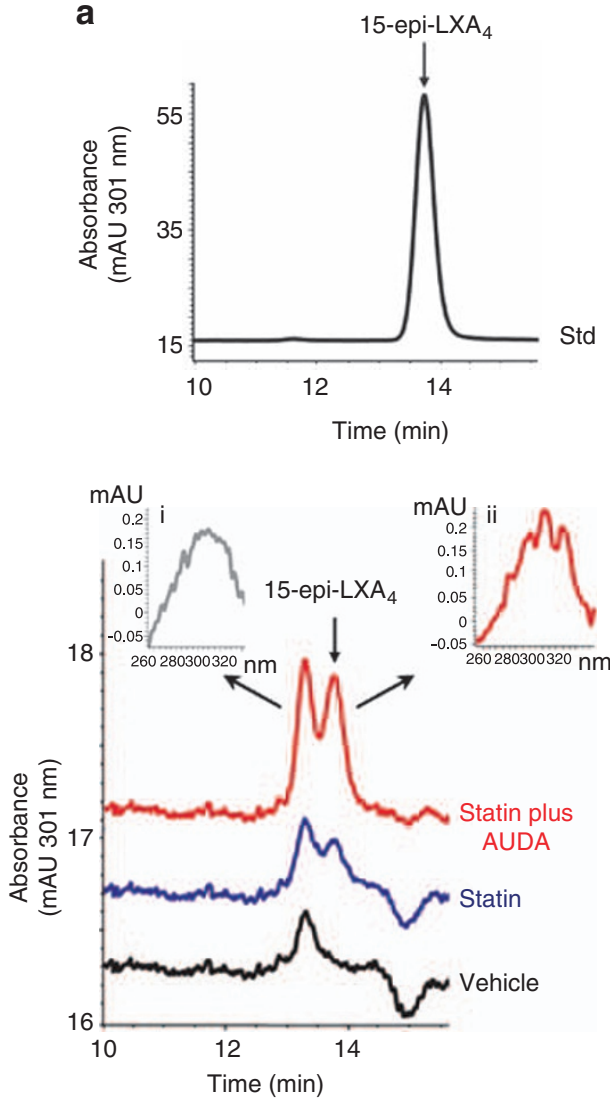

C

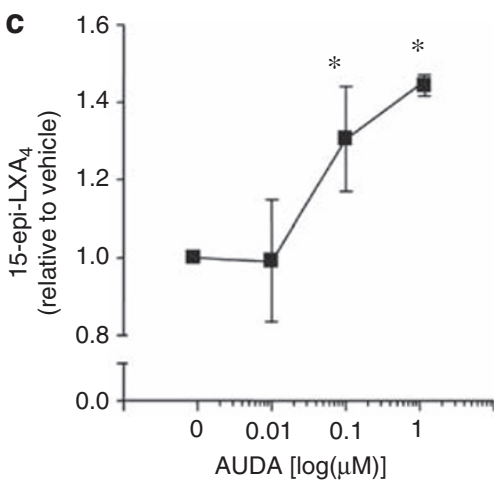

b

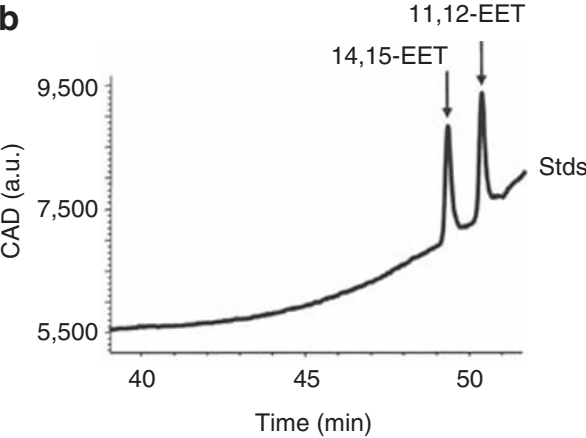

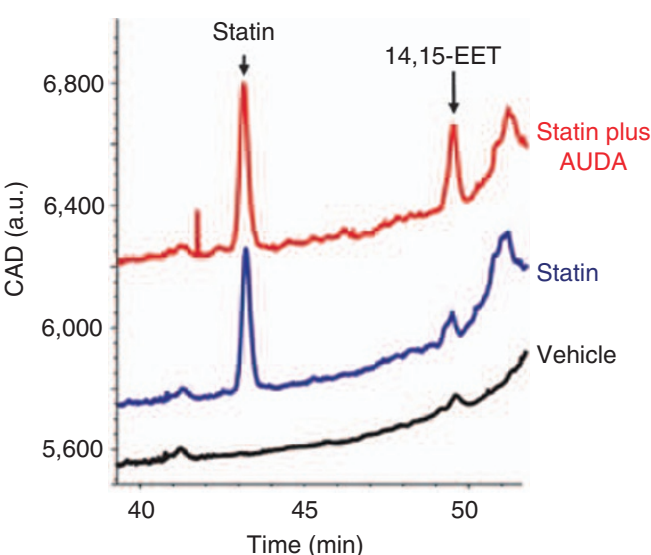

d

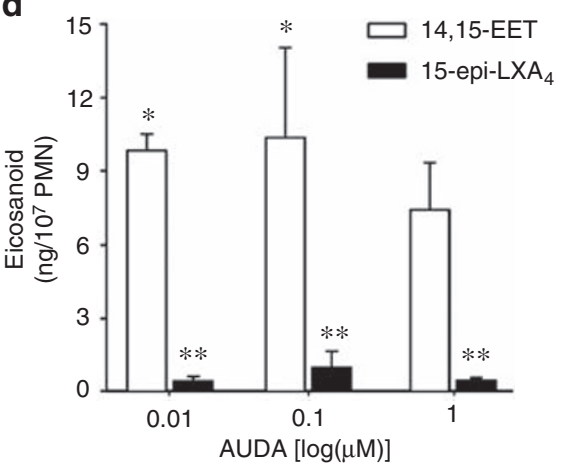

Figure 2 Lovastatin and the soluble epoxide hydrolase (sEH) inhibitor AUDA increase 14,15-epoxyeicosatrienoic acid (14,15-EET) and 15-epilipoxin $\mathrm{A}_{4}$ (15-epi-LXA $_{4}$ formation. Neutrophils (polymorphonuclear leukocytes (PMNs)) were incubated with tumor necrosis factor- $\alpha$ (TNF- $\alpha$ )-exposed Calu-3 cells (5:1, PMNs:Calu-3) in the presence of lovastatin $(1 \mu \mathrm{m})$ and in some cases AUDA $(1 \mu \mathrm{m})$ for $30 \mathrm{~min}\left(37^{\circ} \mathrm{C}\right)$. Lipids were extracted and analyzed by reversed-phase high-performance liquid chromatography (RP-HPLC) using (a) absorbance at $301 \mathrm{~nm}$ (Inset, ultraviolet (UV) spectrum for (i) non-specific materials at $13.3 \mathrm{~min}$ and (ii) for $15-\mathrm{epi}-\mathrm{LXA}_{4}$ at $13.7 \mathrm{~min}$ ) or (b) charged aerosol detector (CAD) (see Methods). Authentic materials are shown in the upper panels. (c,d) Concentration response to AUDA $(0.01-1 \mu \mathrm{m})$ on statin-triggered 15-epi-LXA ${ }_{4}$ formation relative to $(\mathbf{c})$ vehicle and (d) 14,15-EET by TNF- $\alpha$-exposed Calu-3 cells and PMNs. 15-epi-LXA 4 levels were determined using enzyme-linked immunosorbent assay (ELISA). Results are expressed as mean \pm S.e.m. ( $n=3$ independent experiments). ${ }^{*} P<0.05$ relative to no AUDA and ${ }^{* *} P<0.05$ compared with $14,15-\mathrm{EET}$ using analysis of variance (ANOVA).

additional action as a suicide inhibitor of 20-hydroxylase metabolism of eicosanoids. ${ }^{33}$ Incubation of 14,15-EET with recombinant 5-LO confirmed that 14,15-EET is not directly converted to 15 -epi-LXA 4 or other tetraene-containing products. To determine whether the relationship between 14,15-EET and 15-epi$\mathrm{LXA}_{4}$ was bidirectional, PMNs were activated in the presence of 15 -epi-LXA $4(1 \mu \mathrm{M})$, but there was no detectable 14,15-EET generated.

\section{Lovastatin promotes the resolution of acute lung injury by increasing 15-epi-LXA formation in vivo}

To analyze the in vivo effect of the in vitro findings with airway epithelial cells and PMNs, lovastatin $\left(0.2\right.$ or $\left.2 \mathrm{mg} \mathrm{kg}^{-1}\right)$ or vehicle was administered intravenously $15 \mathrm{~min}$ before ALI, and lung leukocyte infiltration was determined $18 \mathrm{~h}$ later during the early resolution phase of this model. Lovastatin was administered in $\mathrm{mg} / \mathrm{kg}$ doses based on its in vivo activity in other murine 

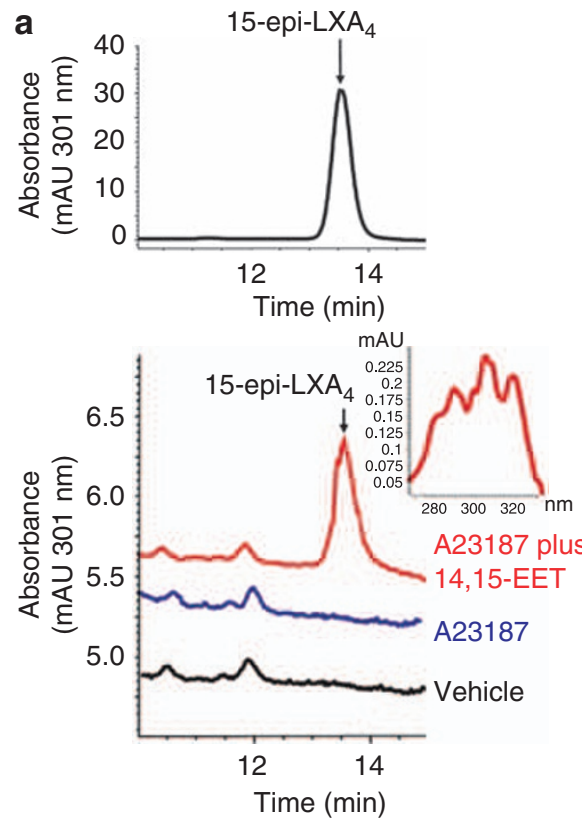

b

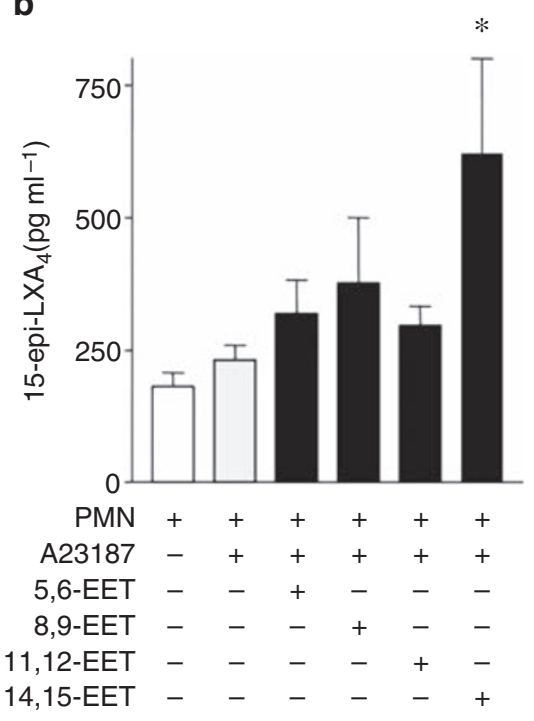

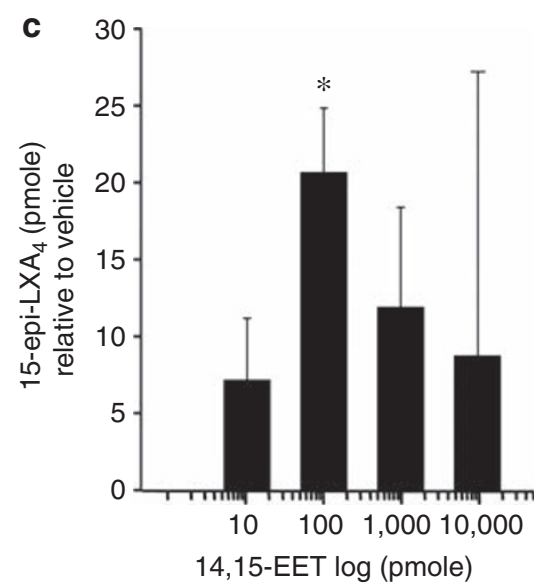

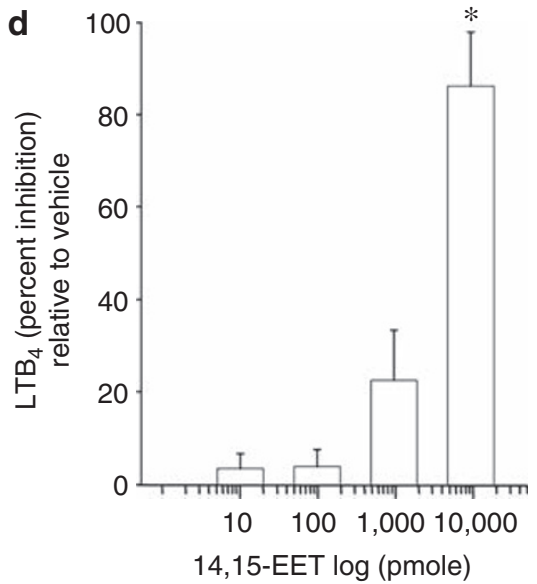

Figure 3 Regiospecific influence of 14,15-epoxyeicosatrienoic acid (14,15-EET) on 15-epi-lipoxin $\mathrm{A}_{4}$ (15-epi-LXA $)$ biosynthesis by activated human polymorphonuclear leukocytes (PMNs). Freshly isolated human PMNs were activated with $A 23187$ in the presence of EETs $(1 \mu \mathrm{M})$ or vehicle $(0.1 \%$ ethanol). After extraction, 15-epi-LXA levels were measured by (a) reversed-phase high-performance liquid chromatography (RP-HPLC; authentic 15-epi-LXA 4 is shown in the upper panel) or (b) enzyme-linked immunosorbent assay (ELISA; see Methods). Concentration response for 14,15-EET $\left(10-10,000\right.$ pmole) on (c) 15 -epi-LXA ${ }_{4}$ and (d) LTB $_{4}$ formation. Values represent the mean \pm s.e.m. for $\geqslant 3$ independent experiments. ${ }^{*} P<0.05$ using Student's $t$-test.

models of lung inflammation. ${ }^{12,34}$ Bronchoalveolar lavage fluids (BALFs) after ALI contained increased numbers of total leukocytes, especially macrophages $(\mathrm{M} \phi)$ and PMNs, compared with BALFs from control animals receiving sterile saline $(0.9 \%)$ (Figure $4 a, b)$. At this time point, lovastatin significantly decreased total BALF cells in a dose-dependent manner. Lowdose lovastatin $\left(0.2 \mathrm{mg} \mathrm{kg}^{-1}\right)$ decreased BALF PMNs, but only the higher dose $\left(2 \mathrm{mg} \mathrm{kg}^{-1}\right)$ significantly decreased both $\mathrm{M} \phi$ and PMNs. Of interest, administration of lovastatin in the absence of acid injury also showed anti-inflammatory properties in blocking PMNs $(P<0.05)$ and increasing $\mathrm{M} \phi$ trafficking related to low-level inflammation associated with the surgical procedure. The protective actions of lovastatin for BALF PMNs were also evident in the lung parenchyma with decreased numbers of cells positive for the PMN marker Ly-6G (Figure 4c). Concomitant with decreased lung leukocyte infiltration at $18 \mathrm{~h}$ after acid injury, lovastatin-treated mice also had significantly higher 15-epi-LXA levels in BALFs $\left(266 \pm 46 \mathrm{pg} \mathrm{ml}^{-1}\right)$ when compared with vehicle $\left(131 \pm 29 \mathrm{pg} \mathrm{ml}^{-1}, P<0.05\right)$ or uninjured, statinexposed animals $\left(76 \pm 34 \mathrm{pg} \mathrm{ml}^{-1}, P<0.05\right)$ (Figure 4d). When tested in parallel, lovastatin's actions on airway leukocytes, in particular on airway PMNs, were similar in magnitude to direct administration of $2 \mathrm{mg} \mathrm{kg}^{-1}$ of 15 -epi-LXA 4 (Figure 4e,f). In addition, lovastatin and 15-epi-LXA 4 yielded additive inhibition of airway inflammation (Figure 4e,f). 14,15-EET also decreased airway PMN trafficking after ALI.

\section{DISCUSSION}

The results presented in this study have identified biosynthetic pathways for statin-triggered 15-epi-LXA formation during cell-cell interactions between human PMNs and airway epithelial cells. Neither cell type alone was independently capable 

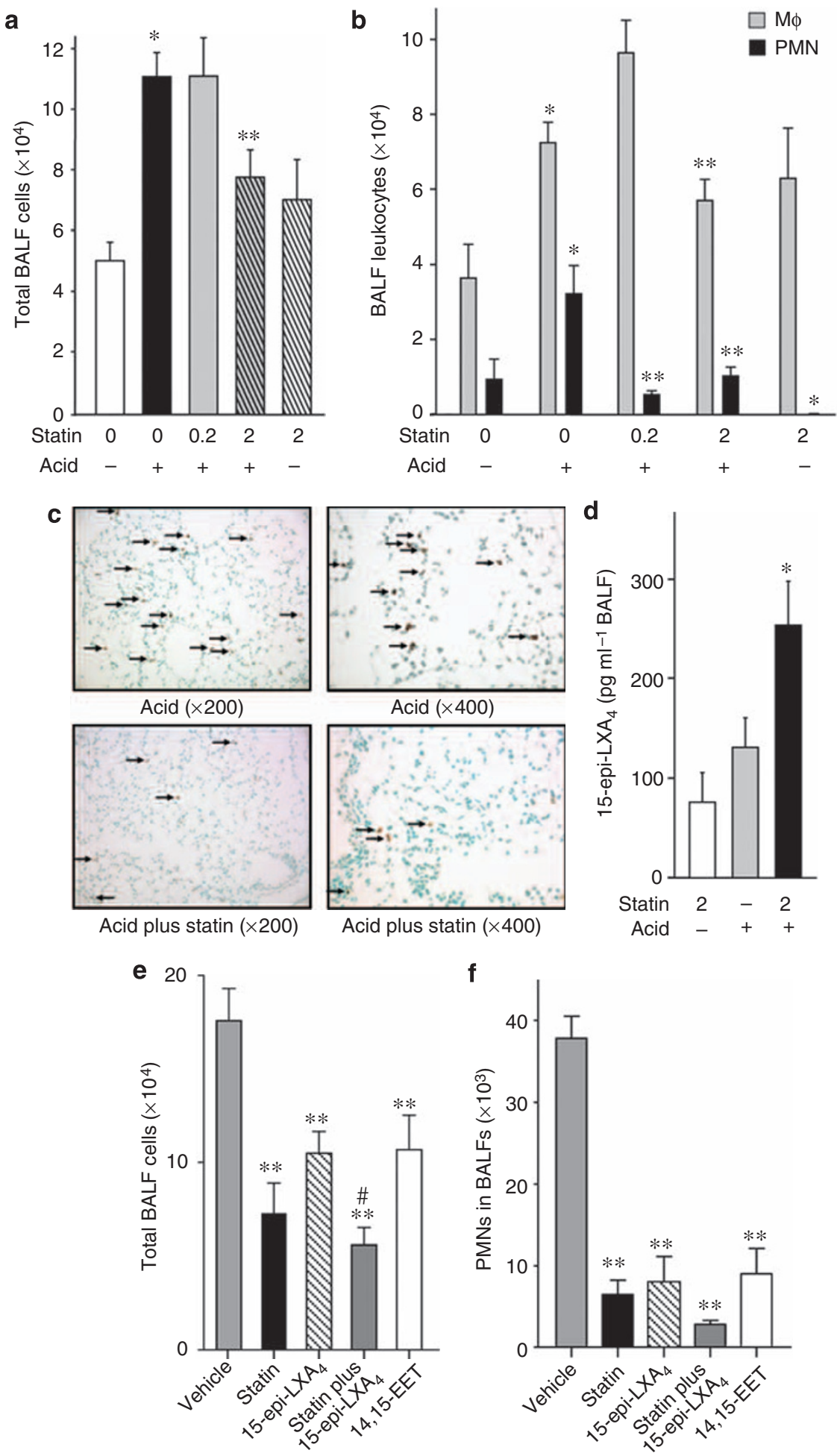

Figure 4 Lovastatin promotes 15-epi-lipoxin $\mathrm{A}_{4}$ (15-epi-LXA $)$ formation in vivo and acute lung injury (ALI) resolution. Lovastatin (0.2 or $2 \mathrm{mg} \mathrm{kg}^{-1}$ Statin) or vehicle $(0.9 \%$ saline) were administered intravenously $15 \mathrm{~min}$ before hydrochloric acid (HCl)-initiated ALI and bronchoalveolar lavage fluids (BALFs) were obtained 18h later. (a) Total BALF cells were enumerated, and (b) the number of BALF macrophages $(\mathrm{M} \phi)$ and neutrophils (polymorphonuclear leukocyte (PMN)) were determined (see Methods). (c) Immunostaining for Ly-6G (1:50 dilution) in murine lung tissue obtained 18h after acid-induced $\mathrm{ALI}$ in the absence (upper panels) or presence of lovastatin (lower panels). PMNs are highlighted by arrows and original magnifications are indicated. (d) 15-epi-LXA 4 levels were determined using enzyme-linked immunosorbent assay (ELISA) in BALFs. For purposes of direct comparison, resolution of lung leukocyte infiltration at $18 \mathrm{~h}$ was determined by monitoring (e) total BALF leukocytes and (f) BALF PMNs in mice administered $\left(2 \mathrm{mg} \mathrm{kg}^{-1}\right.$, intravenous, $100 \mu \mathrm{l})$ lovastatin, 15-epi-LXA $, 14,15$-epoxyeicosatrienoic acid (14,15-EET), the combination of 15-epi-LXA ${ }_{4}$ and lovastatin ( $2 \mathrm{mg} \mathrm{kg}^{-1}$ each), or vehicle ( $1 \%$ ethanol) $15 \mathrm{~min}$ before intratracheal acid. Values represent the mean \pm s.e.m. ( $\geqslant 4$ from at least three independent experiments). ${ }^{\star} P<0.05$ compared with no acid or statin, ${ }^{\star *} P<0.05$ compared with vehicle, and ${ }^{\#} P<0.05$ compared with $15-\mathrm{epi}-\mathrm{LXA}_{4}$ alone. 
of substantial generation of 15-epi-LXA , yet together they collaborated to produce this anti-inflammatory and pro-resolving mediator. Lovastatin's induction of 15 -epi-LXA 4 was shared with simvastatin, but not blocked by the addition of mevalonate, indicating that these statin properties were not solely secondary to inhibition of 3-hydroxy-3-methyl-glutaryl-CoA reductase. In contrast, 15 -epi-LXA 4 production was dependent on LO activity. Unexpectedly, lovastatin also increased 14,15EET formation during PMN-airway epithelial cell interactions, suggestive of decreased sEH activity, and in separate experiments the potent sEH inhibitor AUDA markedly increased 15-epi-LXA 4 production. 14,15-EET showed a regioselective

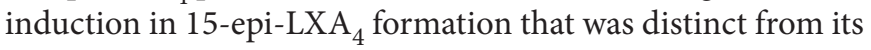
effects on $\mathrm{LTB}_{4}$ generation. 14,15-EET was not a direct substrate for 5-LO-mediated conversion, and hence this CYP450 enzymederived product indirectly altered arachidonic acid metabolism to favor 15-epi-LXA 4 . These cell-cell interactions in vitro were also present in vivo in an experimental model of PMN-airway epithelial cell interactions. Lovastatin dampened acid-initiated ALI in a dose-dependent manner, in particular decreasing PMN

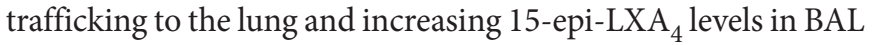
fluids. Exogenous addition of 15 -epi- $\mathrm{LXA}_{4}$ also showed tissue protective actions that were additive with lovastatin's effects. Together, these results are the first to (i) identify statin-triggered 15-epi-LXA 4 generation by human cells, (ii) determine its transcellular biosynthetic routes, (iii) assign roles for CYP450 intermediates in its biosynthesis, and (iv) uncover 15-epi-LXA as an in vivo mechanism for statin's anti-inflammatory actions.

Interactions between PMNs and tissue resident cells are critical to innate immunity. ${ }^{13}$ When damaged by injury or exposed to potential pathogens, mucosal epithelial cells rapidly signal for PMN accumulation and activation to maintain mucosal barrier integrity and host defense. Because PMN anti-microbial effector mechanisms can also have an unintended capacity to damage host tissues, the interplay between PMNs and epithelial cells is tightly regulated to control the intensity of acute mucosal inflammation and prevent extensive tissue damage (reviewed in ref. ${ }^{35}$ ). To this end, counter-regulatory and pro-resolving mediators can stop PMN activation, block the release of epithelial pro-inflammatory mediators, enhance macrophage clearance of apoptotic PMNs, and increase expression of epithelial anti-microbial peptides (reviewed in ref. ${ }^{14}$ ). Transient exposure of human bronchial epithelium to hydrochloric acid upregulates LX receptors and LXs enhance restitution of the injured epithelium and potently block acid-triggered cytokine release and neutrophil transepithelial migration. ${ }^{36}$ By inducing 15-epiLX generation during PMN-epithelial cell interactions, statins initiated the formation of mediators with autocrine anti-inflammatory and pro-resolving actions for these cells. In the presence of statins, epithelial cells exposed to increased inflammation in the form of greater numbers of activated PMNs yielded a dosedependent increase in 15-epi-LXA 4 production. Together, these findings uncovered new roles for transcellular biosynthesis in statin-mediated promotion of pro-resolving mediator generation to counter acute PMN-rich inflammation at mucosal epithelial interfaces.
15-epi-LXs are generated in vivo during inflammation in human disease. ${ }^{37}$ Although CYP450 enzymes can generate $15-R$ hydroxyeicosatetraenoic acid as a biosynthetic intermediate for 15 -epi-LXs, ${ }^{27}$ novel biosynthetic pathways were recently elucidated in rat myocytes in which atorvastatin and/or pioglitazone can trigger atypical interactions between post-translationally modified COX-2 and 5-LO. ${ }^{38}$ Although these findings link statin-mediated generation of 15 -epi-LXA 4 to tissue protection in rat myocardium, ${ }^{24}$ the results presented in this study build on those with rat myocytes to broaden the implication for statintriggered 15-epi-LXA 4 generation to mucosal anti-inflammation with human cells and murine tissues in vivo. Of interest, statins altered CYP450 metabolism of arachidonic acid to increase levels of 14,15 -EET that in turn increased 15 -epi-LXA 4 production by indirect means. Exogenous addition of 14,15-EET to activated human PMNs led to 15 -epi-LXA 4 generation with a bell-shaped dose-response relationship that may have resulted from cytotoxicity at the higher doses of $14,15-\mathrm{EET}$ as $\mathrm{LTB}_{4}$ generation was also inhibited. Whereas 5,6-EET can serve as a direct substrate for platelet lipoxygenase, ${ }^{39}$ evidence presented in this study did not support direct LO conversion of 14,15-EET to epi-LXs. 14,15-EET is the most abundant EET in the lung, ${ }^{40}$ and in this study the influence of 14,15 -EET was regioselective, indicative of specific lipid-protein interactions. In addition, a sEH inhibitor markedly increased both 14,15-EET and 15-epi$\mathrm{LXA}_{4}$ formation. Inhibition of $s \mathrm{EH}$ during acute inflammation can also increase LX formation in vivo. ${ }^{31}$ The exact mechanism for the positive influence of 14,15-EET on LX and 15-epi-LX biosynthesis remains to be elucidated.

Both EETs and LXs show anti-inflammatory actions, but their concentration responses are distinct and target dependent. In nano- to micromolar amounts, EETs can inhibit NF- $\kappa B$, release of pro-inflammatory mediators, and leukocyte adhesion..$^{29,41}$ In sharp contrast, LXs and 15-epi-LXs show these protective actions yet with half-maximal inhibitory concentration in the pico- to nanomolar range and activate specific G-proteincoupled receptors. ${ }^{19,42}$ In this study the results with statins serendipitously uncovered the capacity for EETs to promote 15-epi-LX production. In the presence of lovastatin and the sEH inhibitor AUDA, the generation of EETs and 15-epi-LXA 4 each increased in an approximate 10:1 ratio of 14,15-EET to 15-epi-LXA 4 . In addition, both 14,15-EET and 15-epi-LXA blocked PMN trafficking in vivo in acid-initiated ALI. Thus, these results suggest that the anti-inflammatory actions of EETs may result from endogenous generation of 15-epi-LXs that activate pro-resolving circuits in inflammation.

Statins seem to have particularly beneficial actions in several forms of lung disease. Several observational studies have suggested a link between pre-existing statin use and improved outcomes in ALI and sepsis. ${ }^{43-46}$ Recent data analyses from the Normative Aging Study ${ }^{47}$ revealed that statin use attenuated the decline of lung function in the elderly, including among past and present smokers, and the Veterans Affairs Health Care System database ${ }^{48}$ revealed a protective effect for statins against the development of lung cancer. In addition, statin use is associated with improved allograft function after lung transplantation. ${ }^{49}$ In model systems, statins inhibit 
cigarette smoke-induced emphysema, pulmonary hypertension, and lung inflammation. ${ }^{5,6,12}$ Although less information is available on 15-epi-LX generation in human illness, these mediators were identified in plasma ${ }^{50}$ and in the airways of human subjects with chronic obstructive pulmonary disease. ${ }^{37}$ In this study lovastatin decreased ALI-initiated airway inflammation by triggering an increase in lung 15 -epi-LXA ${ }_{4}$. When administered with lovastatin, 15-epi$\mathrm{LXA}_{4}$ blocked lung inflammation in an additive manner. Together, these findings indicate 15-epi- $\mathrm{LXA}_{4}$ biosynthesis is an endogenous mechanism that underlies statins' protective actions in the lung.

In conclusion, statins initiated the endogenous biosynthesis of 15-epi-LXs during PMN-epithelial cell interactions in vitro and in mucosal inflammation in vivo. Statins promoted 15-epi-LXA generation by decreasing $\mathrm{sEH}$ activity to increase 14,15-EET that influences arachidonate conversion to 15-epi-LXs. Thus, the present results have uncovered endogenous 15-epi-LX generation as a pivotal mechanism involved in statin's known anti-inflammatory actions and suggest that statins can activate resolution circuits in vivo through 15 -epi-LXA biosynthesis. Given the observed clinical benefits for statin therapy, our findings raise the possibility that enhancing 15 -epi-LX production may also enhance the anti-inflammatory actions of statins.

\section{METHODS}

PMN Isolation and Incubations. Fresh venous blood $(\sim 180 \mathrm{ml})$ was obtained from healthy volunteers who had not taken any medication for at least 2 weeks, and PMNs were isolated as in Romano et al. ${ }^{32}$ The protocol was approved by the Partners Healthcare institutional review board and written informed consent was obtained from all subjects. Freshly isolated PMNs were suspended $\left(10 \times 10^{6} \mathrm{PMNs}\right.$ per $\left.\mathrm{ml}\right)$ in phosphate-buffered saline containing $130 \mathrm{mgl}^{-1}$ calcium and $100 \mathrm{mgl}^{-1}$ magnesium. In select experiments, PMNs were exposed (30 min at $\left.37^{\circ} \mathrm{C}\right)$ to 5,6 -EET, 8,9-EET, 11,12 -EET or 14,15 -EET $(0-10 \mu \mathrm{M})$, or 15 -epi-LXA $4(1 \mu \mathrm{M})$, followed by A23187 $\left(5 \mu \mathrm{M}, 15 \mathrm{~min}\right.$ at $\left.37^{\circ} \mathrm{C}\right)$. For incubations with inhibitors, PMNs were exposed $\left(10 \mathrm{~min}\right.$ at $\left.37^{\circ} \mathrm{C}\right)$ to methyl arachidonyl fluorophosphonate $(10 \mu \mathrm{M})$, AA-861 $(20 \mu \mathrm{M})$, 17 -octadecynoic acid $(20 \mu \mathrm{M})$, or vehicle $(0.1 \%$ ethanol) before cell activation. All incubations were stopped with two volumes of cold methanol (2:1, vol:vol) and samples were stored at $-20^{\circ} \mathrm{C}$.

Airway epithelial cell culture and incubations. Human airway epithelial cells (Calu- 3 or A549 cells) were cultured to confluence $\left(\sim 3 \times 10^{6}\right.$ cells), and then exposed (24h) to tumor necrosis factor- $\alpha\left(1 \mathrm{ng} \mathrm{ml}^{-1}\right)$ before the addition of freshly isolated human PMNs $\left(15 \times 10^{6}\right.$ PMNs per $\mathrm{ml})$ for $30 \mathrm{~min}\left(37^{\circ} \mathrm{C}\right)$ in Hanks' balanced salt solution containing calcium and magnesium (Invitrogen, Carlsbad, CA) in the presence or absence of lovastatin hydroxy acid $(1 \mu \mathrm{M}$; Cayman Chemical, Ann Arbor, MI) as in Morimoto et al..$^{34}$ In some experiments, Calu-3 cells were exposed $\left(15 \mathrm{~min}, 37^{\circ} \mathrm{C}\right)$ to nordihydroguaiaretic acid $(5 \mu \mathrm{M})$, mevalonate $(100 \mu \mathrm{M})$, simvastatin $(10 \mu \mathrm{M})$, or AUDA $(0.1-1 \mu \mathrm{M})$ before addition of PMNs. Incubations were stopped with cold methanol (2:1, vol:vol) and samples were then stored at $-20^{\circ} \mathrm{C}$.

Primary NHBE cells (Clonetics-BioWhittaker, San Diego, CA) were maintained in culture for 21 days to obtain a differentiated cell population with a mucociliary phenotype as in Bonnans et al. ${ }^{36}$ Differentiated NHBE cells were exposed to tumor necrosis factor- $\alpha\left(1 \mathrm{ng} \mathrm{ml}^{-1}, 24 \mathrm{~h}\right)$ and then PMNs were added with lovastatin $(1 \mu \mathrm{M})$ or vehicle $(0.1 \%$ ethanol) for $30 \mathrm{~min}\left(37^{\circ} \mathrm{C}\right)$. Incubations were stopped and stored as above.

Lipid mediators. To identify eicosanoids, materials were first extracted with $\mathrm{C}_{18}$ Sep-Pak cartridges (Waters, Milford, MA). ${ }^{17}$ Prostaglandin $\mathrm{B}_{2}$ (100 ng) was added to each sample as an internal standard for extraction recoveries. Materials in the methyl formate eluate (i.e., 15-epi-LXs) were brought to dryness under a gentle stream of $\mathrm{N}_{2}$, resuspended in $1 \mathrm{ml}$ of methanol, and kept at $-20^{\circ} \mathrm{C}$. In all, $30 \%$ of the methyl formate fraction was analyzed using reversed-phase HPLC (Agilent 1100 series; Agilent Technologies, Palo Alto, CA) equipped with an Ultrasphere C18 $(250 \times 4.6 \mathrm{~mm}, 5 \mu \mathrm{m}$; Phenomenex, Torrance, CA) column and coupled to a photodiode array detector (ultraviolet and visible range). In addition, a second HPLC system coupled to a Corona charged aerosol detector (CAD) (ESA, Chelmsford, MA) enabled detection of select lipid mediators in the low-picogram range.

The mobile phase was methanol-distilled $\mathrm{H}_{2} \mathrm{O}$-glacial acetic acid (70:30:1, vol:vol:vol) as phase one $\left(t_{0}-30 \mathrm{~min}\right)$ and a linear gradient with methanol (100\%) as phase two $(30-65 \mathrm{~min})$ at an initial flow rate of $0.5 \mathrm{ml} \mathrm{min}^{-1}\left(t_{0}-30 \mathrm{~min}\right)$ followed by $1 \mathrm{ml} \mathrm{min}^{-1}(30-65 \mathrm{~min})$. The criteria used for identification included retention time, ultraviolet spectra, and charged aerosol detection. 15-epi- $\mathrm{LXA}_{4}$ and $\mathrm{LTB}_{4}$ were monitored using both reversed-phase HPLC and enzyme-linked immunosorbent assay (Neogen, Lexington, $\mathrm{KY}$ ); prostaglandin $\mathrm{B}_{2}$ was monitored using HPLC-diode-array detection and EETs using HPLC-charged aerosol detection as they did not possess specific chromophores.

Reverse transcriptase-PCR. Total RNA was isolated using Trizol reagent (Invitrogen Life Technologies) and residual DNA was removed by DNAse I (Invitrogen Life Technologies). After reverse transcription (Sensiscript, Qiagen, Valencia, CA), PCR was performed using $1 \mu \mathrm{g}$ complementary DNA per reaction with specific primers for human $\mathrm{sEH}$ (40 cycles) (forward: 5'-TGTAAATAGCCCAGAAGAGCCCAG-3', reverse: 5' -ACATCTGAGGAACGAGCACGAAGT-3'); 15-LO-1 (45 cycles) (forward: 5' -CCGACCTCGCTATCAAAGAC-3', reverse: GGATGAC CATGGGCAAGAG-3'); 15-LO-2 (45 cycles) (forward: 5' -TGGACAA TCTGGGCAAGGAGTTCA-3' , reverse: 5' -ATTCAGGAACTG GGAGGCGAAGAA-3'); 5-LO (40 cycles) (forward: 5' -ATCAGGACG TTCACGGCCGAGG-3', reverse: 5' -CCAGGAACAGCTCGTTTT CCTG-3'); 5-LO-activating protein (40 cycles) (forward: $5^{\prime}$-GGCCAT CGTCACCCTCATCAGCG-3', reverse: 5'-GCCAGCAACGGACAT GAGGAACAGG-3'); 12-LO (40 cycles) (forward: 5' -TGGACACTGA AGGCAGGGGCT-3', reverse: 5'-GGCTGGGAGGCTGAATCTGGA-3'); and COX-2 (40 cycles) (forward: $5^{\prime}$-TTCAAATGAGATTGTGGGAAAAT TGCT-3', reverse: 5' -AGATCATCTCTGCCTGAGTATCTT-3'). Human $\beta$-actin was used as internal control. ${ }^{17}$

Acid-initiated acute lung injury. All animal protocols were approved by the Harvard Medical Area Animal Institutional Review Board. Hydrochloric acid $(0.1 \mathrm{~N}, \mathrm{pH} 1.5,50 \mu \mathrm{l}$, endotoxin free; Sigma-Aldrich, St Louis, MO) was selectively instilled into the left mainstem bronchus of anesthetized mice (FVB, male, 10-12 weeks; Charles River Laboratories, Wilmington, MA) through a 24-gauge angiocatheter inserted intratracheally as in Fukunaga et al. ${ }^{17}$ To select animals, lovastatin $\left(0.2\right.$ or $\left.2 \mathrm{mg} \mathrm{kg}^{-1}\right)$, 15-epi-LXA $4\left(2 \mathrm{mg} \mathrm{kg}^{-1}\right), 14,15$-EET $\left(2 \mathrm{mg} \mathrm{kg}^{-1}\right)$, the combination of lovastatin and 15-epi-LXA 4 ( $\mathrm{mg} \mathrm{kg}^{-1}$ each), or vehicle ( $<1 \%$ ethanol) was administered intravenously $(100 \mu \mathrm{l}) 15 \mathrm{~min}$ before hydrochloric acid instillation. At $18 \mathrm{~h}$ after hydrochloric acid instillation, BAL was performed with $2 \times 1 \mathrm{ml}$ phosphate-buffered saline with $0.6 \mathrm{~mm}$ EDTA, and cell-free supernatants $\left(200 \times g, 10 \mathrm{~min}\right.$ at $\left.4^{\circ} \mathrm{C}\right)$ were obtained, and 15 epi-LXA 4 levels were measured using enzyme-linked immunosorbent assay (Neogen). Cells in BALFs were resuspended in phosphate-buffered 
saline and enumerated using hemocytometer. Cytospins were performed by cytocentrifuge (STATspin, Westwood, MA) $(265 \times g)$ and leukocyte differentials were determined after Wright-Giemsa stain (Sigma), counting $\geqslant 200$ cells per slide. Lungs were fixed in immunohistochemistry zinc buffer and paraffin embedded for immunostaining with LY-6G (1:50 dilution).

5-LO incubations. 14,15-EET $(100 \mu \mathrm{M})$ was incubated with $20 \mu \mathrm{g}$ of potato 5-LO (Enzo Life Sciences International, Inc., Plymouth Meeting, $\mathrm{PA}$ ) (at $30^{\circ} \mathrm{C}$ for $10 \mathrm{~min}$ ) in $0.1 \mathrm{M} \mathrm{K}_{2} \mathrm{HPO}_{4}$ buffer ( $\mathrm{pH}$ 6.3). Lipoxygenase activity was measured at 0 and 10 min using a spectrophotometer monitoring absorbance at 301,270 , and $234 \mathrm{~nm}$.

Statistical analysis. Values for eicosanoid levels were analyzed using Student's $t$-test and analysis of variance. Data are presented as the mean \pm s.e.m., and a $P$-value of $<0.05$ was considered significant.

SUPPLEMENTARY MATERIAL is linked to the online version of the paper at http://www.nature.com/mi

\section{ACKNOWLEDGMENTS}

We thank GuangLi Zhu for technical assistance. This work was supported by the NIH Grants HL68669 and P50-DE016191 and by a post-doctoral fellowship from the Spanish Ministerio de Educacion y Ciencia (EX2006-0713).

\section{DISCLOSURE}

C.N. Serhan and B.D. Levy are co-inventors on patents on lipoxins that are assigned to Brigham and Women's Hospital and have been licensed for clinical development and are the subject of consultancies for C.N. Serhan and B.D. Levy. The remaining authors declare no conflict of interest.

C) 2010 Society for Mucosal Immunology

\section{REFERENCES}

1. Jain, M.K. \& Ridker, P.M. Anti-inflammatory effects of statins: clinical evidence, basic mechanisms. Nat. Rev. Drug Discov. 4, 977-987 (2005).

2. Kwak, B., Mulhaupt, F., Myit, S. \& Mach, F. Statins as a newly recognized type of immunomodulator. Nat. Med. 6, 1399-1402 (2000).

3. Mira, E. et al. Statins induce regulatory T cell recruitment via a CCL1 dependent pathway. J. Immunol. 181, 11 (2008).

4. Murphy, D.M. et al. Simvastatin attenuates release of neutrophilic and remodeling factors from primary bronchial epithelial cells derived from stable lung transplant recipients. Am. J. Physiol. Lung. Cell Mol. Physiol. 294, L592-L599 (2008).

5. Takahashi, S. et al. Reversal of elastase-induced pulmonary emphysema and promotion of alveolar epithelial cell proliferation by simvastatin in mice. Am. J. Physiol. Lung Cell Mol. Physiol. 294, L882-L890 (2008).

6. Taraseviciene-Stewart, L. et al. Simvastatin causes endothelial cell apoptosis and attenuates severe pulmonary hypertension. Am. J. Physiol. Lung Cell Mol. Physiol. 291, L668-L676 (2006).

7. Ridker, P.M. et al. Rosuvastatin to prevent vascular events in men and women with elevated C-reactive protein. N. Engl. J. Med. 359, 2195-2207 (2008).

8. Merx, M.W. et al. HMG-CoA reductase inhibitor simvastatin profoundly improves survival in a murine model of sepsis. Circulation 109, 25602565 (2004).

9. Leung, B.P. et al. A novel anti-inflammatory role for simvastatin in inflammatory arthritis. J. Immunol. 170, 1524-1530 (2003).

10. McKay, A., Leung, B.P., McInnes, I.B., Thomson, N.C. \& Liew, F.Y. A novel anti-inflammatory role of simvastatin in a murine model of allergic asthma. J. Immunol. 172, 2903-2908 (2004).

11. Lee, J.H. et al. Simvastatin inhibits cigarette smoking-induced emphysema and pulmonary hypertension in rat lungs. Am. J. Respir. Crit. Care Med. 172, 987-993 (2005).

12. Fessler, M.B. et al. A role for hydroxy-methylglutaryl coenzyme a reductase in pulmonary inflammation and host defense. Am. J. Respir. Crit. Care Med. 171, 606-615 (2005).

13. Ware, L.B. \& Matthay, M.A. The acute respiratory distress syndrome. N. Engl. J. Med. 342, 1334-1349 (2000).
14. Serhan, C.N., Chiang, N. \& Van Dyke, T.E. Resolving inflammation: dual anti-inflammatory and pro-resolution lipid mediators. Nat. Rev. Immunol. 8, 349-361 (2008)

15. Haworth, O. \& Levy, B.D. Endogenous lipid mediators in the resolution of airway inflammation. Eur. Respir. J. 30, 980-992 (2007).

16. El Kebir, D. et al. 15-Epi-lipoxin A4 inhibits myeloperoxidase signaling and enhances resolution of acute lung injury. Am. J. Respir. Crit. Care Med. 180, 311-319 (2009).

17. Fukunaga, K., Kohli, P., Bonnans, C., Fredenburgh, L.E. \& Levy, B.D. Cyclooxygenase 2 plays a pivotal role in the resolution of acute lung injury. J. Immunol. 174, 5033-5039 (2005).

18. Clish, C.B., O'Brien, J.A., Gronert, K., Stahl, G.L., Petasis, N.A. \& Serhan, C.N. Local and systemic delivery of a stable aspirin-triggered lipoxin prevents neutrophil recruitment in vivo. Proc. Natl. Acad. Sci. USA 96, 8247-8252 (1999).

19. Jozsef, L., Zouki, C., Petasis, N.A., Serhan, C.N. \& Filep, J.G. Lipoxin A4 and aspirin-triggered 15-epi-lipoxin A4 inhibit peroxynitrite formation, NFkappa B and AP-1 activation, and IL-8 gene expression in human leukocytes. Proc. Natl. Acad. Sci. USA 99, 13266-13271 (2002).

20. Paul-Clark, M.J., Van Cao, T., Moradi-Bidhendi, N., Cooper, D. \& Gilroy, D.W. 15-epi-lipoxin A4-mediated induction of nitric oxide explains how aspirin inhibits acute inflammation. J. Exp. Med. 200, 69-78 (2004).

21. Canny, G. et al. Lipid mediator-induced expression of bactericidal/ permeability-increasing protein (BPI) in human mucosal epithelia. Proc. Natl. Acad. Sci. USA 99, 3902-3907 (2002).

22. Godson, C., Mitchell, S., Harvey, K., Petasis, N.A., Hogg, N. \& Brady, H.R. Cutting edge: lipoxins rapidly stimulate nonphlogistic phagocytosis of apoptotic neutrophils by monocyte-derived macrophages. J. Immunol. 164, 1663-1667 (2000).

23. Levy, B.D. Myocardial 15-epi-lipoxin A4 generation provides a new mechanism for the immunomodulatory effects of statins and thiazolidinediones. Circulation 114, 873-875 (2006).

24. Birnbaum, Y. et al. Augmentation of myocardial production of 15-epi-lipoxina4 by pioglitazone and atorvastatin in the rat. Circulation 114, 929-935 (2006).

25. Schwab, J.M., Chiang, N., Arita, M. \& Serhan, C.N. Resolvin E1 and protectin D1 activate inflammation-resolution programmes. Nature 447, 869-874 (2007).

26. Claria, J. \& Serhan, C.N. Aspirin triggers previously undescribed bioactive eicosanoids by human endothelial cell-leukocyte interactions. Proc. Natl. Acad. Sci. USA 92, 9475-9479 (1995).

27. Claria, J., Lee, M.H. \& Serhan, C.N. Aspirin-triggered lipoxins (15-epi-LX) are generated by the human lung adenocarcinoma cell line (A549)neutrophil interactions and are potent inhibitors of cell proliferation. Mol. Med. 2, 583-596 (1996).

28. Karara, A., Dishman, E., Blair, I., Falck, J.R. \& Capdevila, J.H. Endogenous epoxyeicosatrienoic acids. Cytochrome P-450 controlled stereoselectivity of the hepatic arachidonic acid epoxygenase. J. Biol. Chem. 264, 19822-19827 (1989).

29. Capdevila, J.H., Harris, R.C. \& Falck, J.R. Microsomal cytochrome P450 and eicosanoid metabolism. Cell. Mol. Life Sci. 59, 780-789 (2002).

30. Moghaddam, M.F., Grant, D.F., Cheek, J.M., Greene, J.F., Williamson, K.C. \& Hammock, B.D. Bioactivation of leukotoxins to their toxic diols by epoxide hydrolase. Nat. Med. 3, 562-566 (1997).

31. Schmelzer, K.R., Kubala, L., Newman, J.W., Kim, I.H., Eiserich, J.P. \& Hammock, B.D. Soluble epoxide hydrolase is a therapeutic target for acute inflammation. Proc. Natl. Acad. Sci. USA 102, 9772-9777 (2005).

32. Romano, M., Chen, X.S., Takahashi, Y., Yamamoto, S., Funk, C.D. \& Serhan, C.N. Lipoxin synthase activity of human platelet 12-lipoxygenase. Biochem. J. 296 (Part 1), 127-133 (1993).

33. Shak, S., Reich, N.O., Goldstein, I.M. \& Ortiz de Montellano, P.R. Leukotriene B4 omega-hydroxylase in human polymorphonuclear leukocytes. Suicidal inactivation by acetylenic fatty acids. J. Biol. Chem. 260, 13023-13028 (1985).

34. Morimoto, K. et al. Lovastatin enhances clearance of apoptotic cells (efferocytosis) with implications for chronic obstructive pulmonary disease. J. Immunol. 176, 7657-7665 (2006).

35. Leitch, A.E., Duffin, R., Haslett, C. \& Rossi, A.G. Relevance of granulocyte apoptosis to resolution of inflammation at the respiratory mucosa. Mucosal. Immunol. 1, 350-363 (2008).

36. Bonnans, C., Fukunaga, K., Levy, M.A. \& Levy, B.D. Lipoxin A(4) regulates bronchial epithelial cell responses to acid injury. Am. J. Pathol. 168, 1064-1072 (2006) 
37. Vachier, I. et al. Severe asthma is associated with a loss of $L X 4$, an endogenous anti-inflammatory compound. J. Allergy Clin. Immunol. 115, 55-60 (2005).

38. Ye, Y. et al. Phosphorylation of 5-lipoxygenase at ser523 by protein kinase a determines whether pioglitazone and atorvastatin induce proinflammatory leukotriene B4 or anti-inflammatory 15-epi-lipoxin a4 production. J. Immunol. 181, 9 (2008).

39. Balazy, M. Metabolism of 5,6-epoxyeicosatrienoic acid by the human platelet. Formation of novel thromboxane analogs. J. Biol. Chem. 266, 23561-23567 (1991).

40. Zeldin, D.C. et al. The rabbit pulmonary cytochrome P450 arachidonic acid metabolic pathway: characterization and significance. J. Clin. Invest. 95, 2150-2160 (1995).

41. Morin, C., Sirois, M., Echave, V., Gomes, M.M. \& Rousseau, E. EET displays anti-inflammatory effects in TNF-alpha stimulated human bronchi: putative role of CPI-17. Am. J. Respir. Cell. Mol. Biol. 38, 192-201 (2008).

42. Fierro, I.M. et al. Lipoxin A4 and aspirin-triggered 15-epi-lipoxin A4 inhibit human neutrophil migration: comparisons between synthetic 15 epimers in chemotaxis and transmigration with microvessel endothelial cells and epithelial cells. J. Immunol. 170, 2688-2694 (2003).

43. Almog, Y. et al. Prior statin therapy is associated with a decreased rate of severe sepsis. Circulation 110, 880-885 (2004).
44. Irish Critical Care Trials G. Acute lung injury and the acute respiratory distress syndrome in Ireland: a prospective audit of epidemiology and management. Crit. Care 12, R30 (2008).

45. Kruger, P., Fitzsimmons, K., Cook, D., Jones, M. \& Nimmo, G. Statin therapy is associated with fewer deaths in patients with bacteraemia. Intensive Care Med. 32, 75-79 (2006).

46. Liappis, A.P., Kan, V.L., Rochester, C.G. \& Simon, G.L. The effect of statins on mortality in patients with bacteremia. Clin. Infect. Dis. 33, 1352-1357 (2001).

47. Alexeeff, S.E., Litonjua, A.A., Sparrow, D., Vokonas, P.S. \& Schwartz, J. Statin use reduces decline in lung function: VA Normative Aging Study. Am. J. Respir. Crit. Care Med. 176, 742-747 (2007).

48. Khurana, V., Bejjanki, H.R., Caldito, G. \& Owens, M.W. Statins reduce the risk of lung cancer in humans: a large case-control study of US veterans. Chest 131, 1282-1288 (2007).

49. Johnson, B.A., Iacono, A.T., Zeevi, A., McCurry, K.R. \& Duncan, S.R. Statin use is associated with improved function and survival of lung allografts. Am. J. Respir. Crit. Care Med. 167, 1271-1278 (2003).

50. Chiang, N., Bermudez, E.A., Ridker, P.M., Hurwitz, S. \& Serhan, C.N. Aspirin triggers antiinflammatory 15 -epi-lipoxin A4 and inhibits thromboxane in a randomized human trial. Proc. Natl. Acad. Sci. USA 101, 15178-15183 (2004). 